\section{Crystal Structure for Approximate Optical Invisibility Cloaking Proposed}

Electromagnetic cloaking of materials has long been fantasized in science fiction tales, but has recently been shown to be theoretically possible. Experimentally, limited forms of cloaking have been realized in the microwave region of the spectrum. Significant work is underway to investigate approaches to cloaking in the optical region of the spectrum. D. Xiao and $\mathrm{H}$. Johnson, of the University of Illinois at Urbana-Champaign, propose a photonic crystal structure capable of approximate cloaking in the optical regime. Their calculations, reported in the April 15 issue of Optics Letters (p. 860), show that anisotropy in an axisymmetric silicon photonic crystal can serve as a waveguide to bend light around an object.

Xiao and Johnson develop a cloaking schema based upon a perfectly layered silicon and air structure that provides approximate cloaking in the optical regime. As opposed to an ideal cloak, a refractive index matched material, this approximate scheme uses a multiply layered structure to
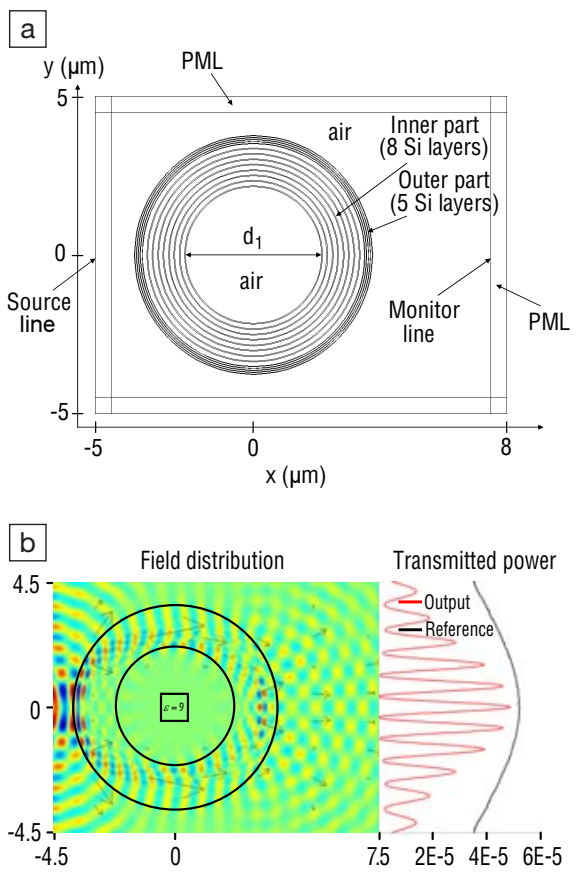

Figure 1. (a) Illustration of the axisymmetric silicon cloaking device.

(b) Demonstration of the magnetic field and power distribution of the device in response to a Gaussian beam. The red lines illustrate the output with the cloaking device and the black is the reference system without the object. Reprinted with permission from Optics Letters 33 (8) (April 15, 2008) p. 860. (C)2008 Optical Society of America. guide the light around an obstacle and prevent it from entering the object, as illustrated in Figure 1. The photonic band structure of the proposed axisymmetric crystal structure in silicon results in a tangential index of refraction of 1.13 and a radial index of 2.07 , resulting in double action as both waveguide and waveblock.

In order to establish a baseline for the efficacy of the cloak, the researchers conducted numerical experiments to compare the optical properties of the proposed cloak with those of an ideal cloak, both illuminated with a Gaussian beam of light. They used these simulations to predict the sensitivity of the cloak to the ratio of particle size to wavelength. Their analysis indicates that the index matched cloaking functions well when the object size is less than $0.05 \%$ of the wavelength of the incident light, but that these cloaks are very sensitive to material perturbations and fail as object size-to-wavelength ratio increases. Though the cloak proposed here is approximate, these results show a potential way to engineer a cloaking device on a larger scale, said the researchers.

ARTHUR FELDMAN

\section{Superlattices of $\mathrm{PbTiO}_{3} / \mathrm{SrTiO}_{3}$ Exhibit Improper Ferroelectricity}

As materials researchers gain increasingly fine control over epitaxial thin film growth, they often find that unexpected properties emerge at the interface between compounds. A new discovery in this type of "interface engineering" is improper ferroelectricity in superlattices of $\mathrm{PbTiO}_{3}$ and $\mathrm{SrTiO}_{3}$ as reported in the April 10 issue of Nature (DOI: 10.1038/nature06817; p. 732). E. Bousquet of the University of Liège, M. Dawber of the University of Geneva, and their co-workers have used a combination of experimental and computational techniques to demonstrate the emergence of improper ferroelectricity in short period superlattices of $\mathrm{PbTiO}_{3} / \mathrm{SrTiO}_{3}$, governed by the interface between the films. The superlattices have a very large dielectric constant that is roughly independent of temperature and a sizable polarization.

The researchers used density functional calculations to determine the structural ground state. Bulk $\mathrm{PbTiO}_{3}$ is polar with a ferroelectric (FE) structural distortion, while bulk $\mathrm{SrTiO}_{3}$ is nonpolar with an antiferrodistortive (AFD) distortion in which the oxygen octahedra in successive (001) layers rotate in opposite directions. When layered in a superlattice, however, the behavior changes. In superlattices of $\mathrm{PbTiO}_{3} / \mathrm{SrTiO}_{3}$ unit cell periodicity of $1 / 1$, the researchers found a polar ground state in which FE and AFD distortions coexist. The energy profile as a function of polarization reveals a term in the Landau expansion of the free energy that is linear in polarization, indicative of improper ferroelectricity. In superlattices with larger periodicity the improper ferroelectricity gives way to regular ferroelectricity because the FE and AFD modes only coexist at the interface, with the AFD distortions disappearing rapidly in the bulk-like segments.

Bousquet and co-workers confirmed this behavior experimentally. They grew $\mathrm{PbTiO}_{3} / \mathrm{SrTiO}_{3}$ superlattices of $9 / 3$ and $2 / 3$ periodicities by off-axis magnetron sputtering on (001) $\mathrm{SrTiO}_{3}$ substrates and measured structural distortion, dielectric properties, and polarization. They found that the polarization, octahedral tilting, and tetragonality of the superlattices all scale with temperature in the manner of a regular ferroelectric for the $9 / 3$ samples but scale like an improper ferroelectric, as described by Landau theory, in the 2/3 superlattices. The dielectric susceptibility follows a Curie-Weiss law for the $9 / 3$ sample in the typical manner for a ferroelectric, but remains essentially constant at all temperatures for the $2 / 3$ sample, with a small step at the transition temperature of $500 \mathrm{~K}$. The researchers conclude that improper ferroelectricity is evident in the $2 / 3$ superlattices and, with a polarization of $11 \mu \mathrm{C} \mathrm{cm}^{-2}$ and a dielectric constant of $\sim 600$ at room temperature, this is an appealing material for technological applications. The researchers said that the coupling of instabilities at the interfaces in artificially layered structures might be transferable to other types of oxides, and could be a particularly interesting strategy in the emerging domain of multiferroics.

ALISON HATT

\section{Hyperfine Interactions in Graphene Calculated from First Principles}

The approach of current silicon-based electronics technology toward its intrinsic limitations has motivated industrial and academic researchers to seek alternate computing paradigms. Spintronics and quantum computing, which use electron spins for operating information, are recognized as the most promising approaches to extend the limits of current chargebased electronics. However, these novel approaches require novel materials. In particular, the problem of electron spin relaxation and decoherence can be solved by using carbon materials, such as graphene and carbon nanotubes. Spin-orbit interactions that couple electrons to phonons are intrinsically weak in these materials. Another source of spin relaxation and decoherence is the interaction with nuclear spins, the so-called hyperfine interaction. 
The nuclei of ${ }^{12} \mathrm{C}$ isotope, which is dominant in carbon materials, has spin zero. However, the ${ }^{13} \mathrm{C}$ isotope, which accounts for $\sim 1 \%$ of carbon nuclei, has spin $1 / 2$. Good understanding of hyperfine interactions and the ability to control them are thus required to overcome spin relaxation and decoherence. Recently, O.V. Yazyev at the Swiss Federal Institute of Technology (EPFL) in Lausanne, Switzerland, used accurate first principles calculations to show that hyperfine interactions in graphene fragments can be accurately described in terms of only the local distribution of low-energy electron spins and the local atomic structure.

As reported in the April issue of Nano Letters (DOI: 10.1021/nl072667q; p. 1011), Yazyev applied all-electron density functional theory implemented in a commercially available quantum chemistry software package. This approach has previously been shown to predict hyperfine coupling constants in excellent agreement with experimental results. Yazyev calculated hyperfine coupling constants for ${ }^{13} \mathrm{C}$ nuclear spins in a dozen electron- and hole-doped graphene fragments $\sim \mathrm{nm}$ in size, providing a database containing over 200 coupling values. Yazyev found that the isotropic (Fermi contact) and dipolar hyperfine couplings in the graphene structures are about equally important. Their magnitudes are significant but generally smaller than those in materials comprised of heavier elements, such as gallium arsenide. Both Fermi contact and dipolar hyperfine coupling constants (for ${ }^{13} \mathrm{C}$ and other nuclear spins from substitution impurities and edges) were fitted to a simple expression composed of terms that account for onsite and nearest-neighbor conduction electron spin populations. Yazyev said that the parameters thereby obtained provide a tool for predicting the hyperfine interactions in arbitrary $\mathrm{sp}^{2}$ carbon nanostructures. This will allow the engineering of nanoscale devices with optimal spin relaxation and decoherence times. Yazyev further said that "the 'contaminant' nuclear spins, e.g. the spins of protons at the edges of graphene nanostructures, have to be considered seriously because their hyperfine couplings were found to be surprisingly high. A chemical way for decoupling these spins has to be devised in [the] future."

\section{STEVEN TROHALAKI}

\section{Gradiently Alloyed $\mathrm{Zn}_{x} \mathrm{Cd}_{1-x} \mathrm{~S}$ Colloidal PL QDs Developed by One-Pot Synthesis}

Colloidal photoluminescent (PL) semiconductor nanocrystals have attracted significant attention over the past decade from the viewpoints of both fundamental science as well as potential applications. When the sizes of the spherical semiconductor nanocrystals become less than or comparable to that of a photogenerated exciton in bulk materials, quantum confinement effects become operative. Quantum dots (QDs) can absorb and then re-emit light in spectral regions that are not covered by their corresponding bulk materials. Now K. Yu and co-workers at the National Research Council of Canada have introduced an innovative synthesis method for three-component QDs that enhances control of blue emission by creating gradients in alloy composition.

Ternary alloy QDs have opened the way to bandgap engineering with stoichiometry as well as size. The research team proposed that two well-known binary QDs, CdS and ZnS with bulk bandgaps of $2.48 \mathrm{eV}$ and $3.7 \mathrm{eV}$ as well as with zincblende lattice constants at room temperature of $5.835 \AA$ and $5.406 \AA$, should provide a useful blend with high blue emission efficiency owing to their low lattice mismatch. Ideally, a gradient ternary ZnCdS QD should have a Zn-rich outer layer for efficient blue emission as well as low toxicity.

As reported in the April 3 issue of the Journal of Physical Chemistry C (DOI: 10.1021/jp710852q; p. 4908), the researchers developed an efficient approach to the synthesis of high-quality $\mathrm{ZnCdS}$ QDs with Cd-rich inner cores and Zn-rich outer shells. The approach features synthetic reproducibility and easy scale-up. Emphasizing "green," environmentally friendly synthesis by using air-stable chemicals, such as zinc stearate, cadmium acetate dihydrate, and elemental sulfur, the researchers monitored the temporal evolution of the optical properties of the resulting growing QDs, and systematically characterized the QD structure and composition by solid-state nuclear magnetic resonance (NMR), x-ray photo-electron spectroscopy, powder x-ray diffraction, and transmission electron microscopy. The solid-state NMR included cross polarization (CP) and dipolar dephased cross polarization.

The trick exploited by the research team was to vary reactivity during the QD synthesis to form the composition gradients. A Cd-rich core is initially formed, as evinced by the redshifting of the bandgap absorption and emission in the early growth stage. With proper choice of reactants and concentrations, there is a gradual formation of $\mathrm{Zn}$-rich outer layers because of the depletion of $\mathrm{Cd}$ in the reaction medium. The outermost layer can be made to consist of $\mathrm{ZnS}$ with little cadmi-

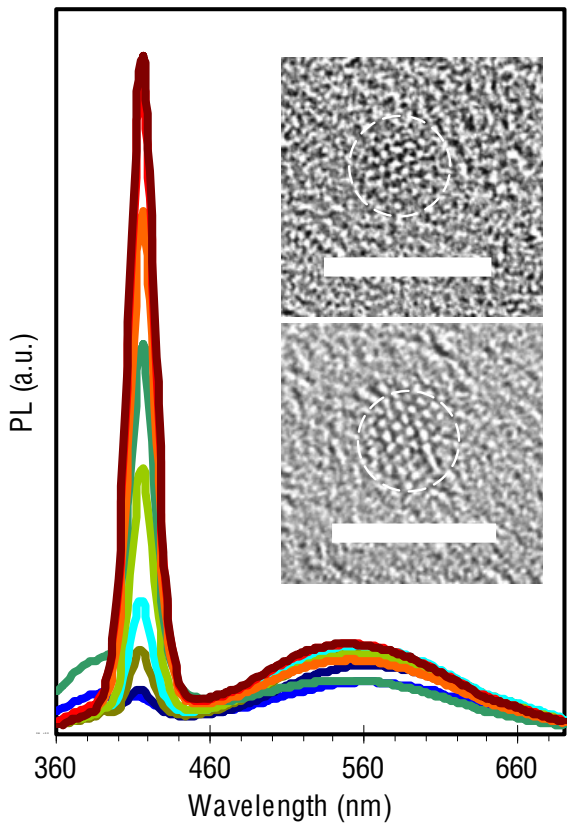

Figure 1. Emission spectra (excitation wavelength $350 \mathrm{~nm}$ ) of the growing ZnCdS nanocrystals from the synthetic batch with a feed molar ratio of $3 \mathrm{Zn}$-to$1 \mathrm{Cd}$-to-4S. The colors represent different growth periods at $240^{\circ} \mathrm{C}$ in minutes (1-180 min.); "fixation" of both the bandgap absorption (at $402 \mathrm{~nm} \pm 1 \mathrm{~nm}$ ) and emission (at $415 \mathrm{~nm} \pm 1 \mathrm{~nm}$ ) is pronounced. The inset transmission electron micrographs represent the nanocrystals with 8-min. (top) and 180-min. (bottom) growth periods, with a scale bar of $5 \mathrm{~nm}$. Reprinted with permission from the Journal of Physical Chemistry C 112 (13) (April 4, 2008) p. 4908. (C2008 American Chemical Society.

um. Such an accumulation of $\mathrm{ZnS}$ in the outer layers directly leads to an effective core-shell structure, which coats and protects the CdS-rich core, giving rise to the observed "fixation" of bandgap absorption and emission, as shown in Figure 1.

The researchers tuned the feed $\mathrm{Zn}$-toCd-to-S stoichiometry to form high-quality $\mathrm{Zn}_{x} \mathrm{Cd}_{1-x} \mathrm{~S}$ QDs exhibiting sharp exciton absorptions, narrow PL emissions, and high PL quantum yield up to $23 \%$. With relatively high $\mathrm{Zn}$ and $\mathrm{S}$ feed amounts, the resulting gradiently structured $\mathrm{ZnCdS}$ QDs exhibited superior photostability against UV irradiation and relatively long shelf time in common organic solvents as compared to that of the binary counterparts. The enhanced UV resistance and storage stability of the nanocrystals can be explained by the "coating" effect of the Znrich region. In the later growth stage, $\mathrm{Zn}$ diffuses into the inner CdS-rich core, leading to a blueshifting of the bandgap.

Solid-state magic angle spinning (MAS) 